\title{
Measurement and Visualization of Cell Thickness and Volume by Transmission-Through-Dye Microscopy
}

\author{
Michael A. Model
}

\section{Department of Biological Sciences, Kent State University, Kent, Ohio, USA}

With only a slight modification, standard brightfield imaging can be used for quantitative display of cell surface topography. To achieve that, the sample is placed in a shallow gap about $30 \mu \mathrm{m}$ deep. Such a gap can be formed between two pieces of glass (coverslip and a slide) kept apart by a small amount of silicone grease. Alternatively, an attachment to a condenser with a horizontal watertight window at the bottom can be built for an inverted microscope; it is lowered into a Petri dish to bring the window close to the cells, thus creating a temporary narrow gap between the cells and the window. Cells are kept in their normal environment, except that a nontoxic dye with a strong absorption in the visible range is added to the medium. We usually use food colorant Acid Blue 9, which has a large extinction coefficient at $630 \mathrm{~nm}$ and at moderate concentrations (0.5-1\%) does not affect cell viability and functioning. Because cells displace the dye, the depth of the absorbing layer is complementary to cell thickness; when such a sample is imaged in transmitted light at the wavelength of maximal dye absorption, the resulting contrast reflects cell thickness (Fig. 1A). To convert intensity $I$ to thickness $h$ one has to apply logarithmic transformation to the image and correct it for the background level; for quantitative information, the result is additionally divided by the absorption coefficient $\alpha$ of the liquid (Eq. 1). Quite often, it is cell volume, rather than thickness, that is of primary interest [1]; naturally, from the knowledge of cell thickness at every point, the volume can be easily computed as well.

$$
h=\frac{\operatorname{lnI}}{\alpha}-\frac{\operatorname{lnI} b k g}{\alpha}
$$

We refer to this imaging method as transmission-through-dye (TTD) microscopy [2]. A typical example of a TTD image of cultured cells is shown in Fig. 1B. At very high concentrations of the dye, the vertical resolution can be brought to a low-nanometer range. Such concentrations would be toxic for most live eukaryotic cells but can be used with formaldehyde-fixed cells (Fig. 1C) or bacteria. But even at concentrations that can be safely used with living cells, the vertical resolution can substantially exceed the diffraction limit. The accuracy of thickness measurements by TTD imaging has been verified using spherical beads and confocal scanning. Dead cells with leaky plasma membranes appear darker than the background and are easily distinguishable from intact cells.

The method can be realized on any widefield microscope with only a bandpass filter added anywhere in the optical path or on a laser scanning microscope in transmission mode; either high- or low-power objectives can be used. TTD imaging is compatible with fluorescence or DIC and is gentle on cells because they are not exposed to high-intensity light. Importantly, the results are not affected by possible instability in the light source because both the cells and the background will be identically affected by fluctuations.

The main limitation of TTD is the limited range of thicknesses that can be displayed in a single image. Sometimes, one has to collect two images of the same field to have the thickest and the thinnest cells well resolved. The other potential problem is the contrast of brightfield origin (Becke lines) that may contaminate TTD images. Becke lines develop at steep cell boundaries or around inclusions with a 
different refractive index. When imaging severely dehydrated apoptotic cells, interference from Becke lines can become significant; fortunately, their effect on volume measurements is usually small and can be further corrected if necessary by taking a control image at a nonabsorbing wavelength [2].

Applications of the method can be diverse. Our research mostly focuses on cell volume regulation in apoptosis [3], where cells undergo characteristic shrinkage. Likewise, cell volume is an important readout in investigations of water and ion transport. In quantitative fluorescence work, the knowledge of cell volume allows conversion of the total intracellular fluorophore (as measured by widefield microscopy) into its concentration. High-resolution imaging of the cell surface can be used for studies of the cytoskeleton; although the high vertical resolution is partly undermined by diffraction-limited lateral resolution, the latter can be improved by additional image processing. By loading cells with a red fluorescent marker, damage to the cell membrane can be detected and possibly quantified in the presence of Acid Blue 9, which quenches red fluorescence by direct contact. We are currently working on combining TTD with quantitative phase microscopy [4] to measure the refractive index, which can be used to assess cell hydration status (for example, the loss of water in apoptotic cells). Apart from specific applications, TTD adds strong contrast to transparent samples and can sometimes reveal features that may escape detection with phase contrast or DIC. The method does not have to be limited to biological samples, and its use in manufacturing quality control has been suggested [5].

[1] F Lang, GL Busch, M Ritter, H Völkl, S Waldegger, E Gulbins and D Häussinger, Physiol Rev. 78 (1998), p.247.

[2] MA Model, Microsc. Today, 20 (2012), p.32.

[3] NR Kasim, K Kuželová, A Holoubek and MA Model, Apoptosis (2013) doi: 10.1007/s10495-0130804-Z

[4] G Popescu, "Quantitative phase imaging of cells and tissue", (2011) (McGraw-Hill, New York).

[5] JCM Antón, J Alonso, Pedrero JA and JA Quiroga, Optics Express, 20 (2012), p.28631.

[6] The study was supported by NIH grant 1R15GM186816-01A1 and Ohio Board of Regents. The author gratefully acknowledges the important contributions of NR Kasim, M Pelts, JL Rees, and AK Khitrin to this work.
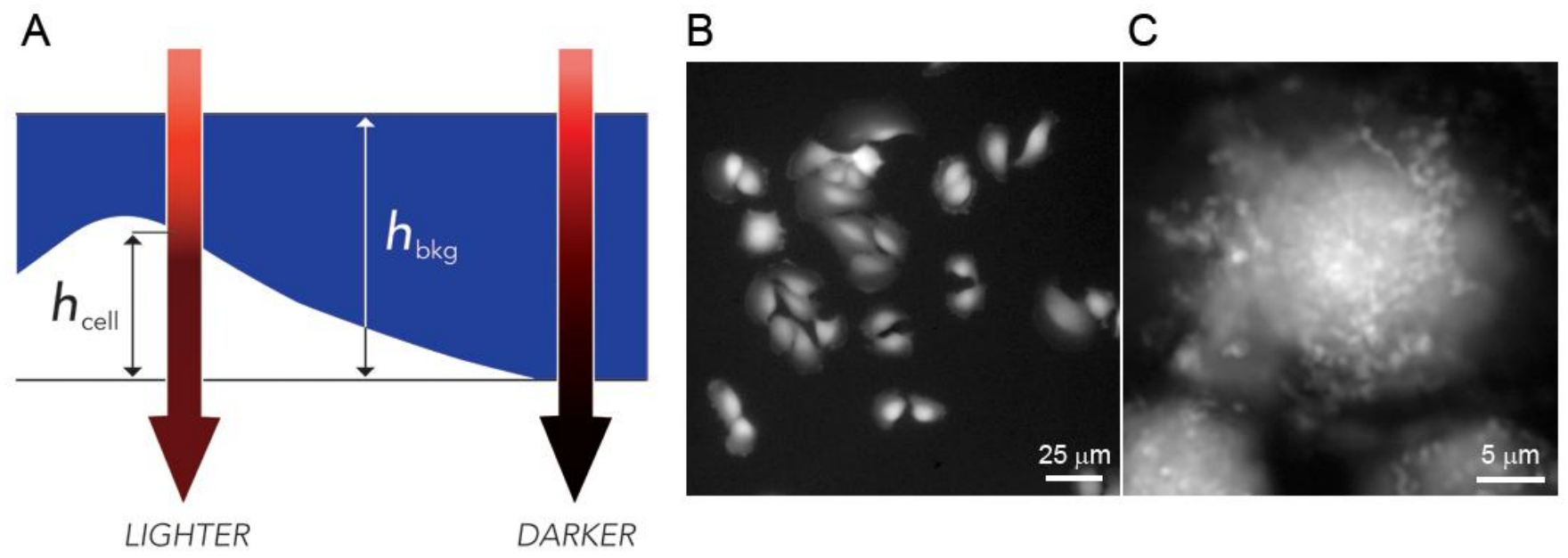

Figure 1. (A) Contrast generation in TTD imaging. (B) Live T24 cells. (C) The surface of a formaldehyde-fixed T24 cell at high resolution. 